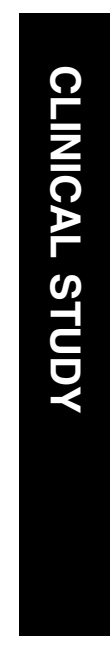

\title{
Long-term intraocular pressure control of eyes that developed encapsulated blebs following trabeculectomy
}

${ }^{1}$ Department of Ophthalmology, University of Campinas, Brazil

${ }^{2}$ Department of Ophthalmology, University of São Paulo, Brazil

${ }^{3}$ Department of Ophthalmology, Federal University of Uberlândia, Brazil

Correspondence: VP Costa, Department of Ophthalmology, University of Campinas, Rua Bauru, 40, Pacaembu, São Paulo, SP 01248-010, Brazil Tel/Fax: + 551138659630 E-mail:vp.costa@ uol.com.br

Received: 24 August 2004 Accepted: 15 February 2005 Published online: 1 April 2005

The authors have no proprietary interest on any substance or instrument mentioned in this study

This study was presented at the 2004 ARVO Meeting in Fort Lauderdale, USA

\begin{abstract}
Purpose To evaluate the long-term intraocular pressure (IOP) control of eyes that developed an encapsulated bleb (EB) following trabeculectomy.

Methods Between 1994 and 1995, 25 eyes developed EBs and were randomized to medical treatment or needling without adjunct antimetabolites. Among the 25 patients who developed an EB, 21 were followed for at least 6 months. A control group of 21 consecutive eyes, which underwent trabeculectomy during the same period and that did not develop EBs was retrospectively selected. Success was defined as IOP $<21 \mathrm{mmHg}$ with or without medications. Kaplan-Meier survival analysis was performed to compare the groups. Results Among the 21 eyes that developed EBs, 12 (57\%) had undergone transconjunctival needling and nine $(43 \%)$ had received medical treatment. Mean follow-ups were $30.0 \pm 14.0$ months, $33.3 \pm 18.5$ months, and $37.4 \pm 2.6$ months for the needling, medical treatment, and control groups, respectively $(P=0.19)$. Kaplan-Meier survival curves demonstrated that the control group showed a significantly lower chance of failure than both the needling and the medical treatment groups $(P<0.0001)$.

Conclusion Encapsulated blebs may be associated with an increased risk for surgical failure.
\end{abstract}

Eye (2006) 20, 304-308. doi:10.1038/sj.eye.6701865; published online 1 April 2005

Keywords: glaucoma, trabeculectomy, failure encapsulated bleb
VP Costa ${ }^{1,2}$, ES Arcieri ${ }^{1,3}$ and TG Freitas ${ }^{1}$

\section{Introduction}

Encapsulated blebs (EBs), also known as Tenon's cysts, are highly elevated, localized blebs, with prominent surface vessels and a patent sclerostomy on gonioscopy. They normally occur 2-4 weeks following filtering surgery and are frequently associated with increased intraocular pressures (IOPs). ${ }^{1-5}$ Different treatment modalities have been suggested to reduce the IOP of eyes with EBs, including the use of aqueous humor suppressors, ${ }^{6-10}$ digital massage, ${ }^{6-10}$ transconjunctival needling, ${ }^{11-14}$ and surgical revision of the bleb. ${ }^{11,12,15}$

Medical treatment with aqueous humor suppressors has been shown to be successful in controlling the IOP immediately after the development of an EB. ${ }^{11-13,15}$ Some have reported disappointing results when needling is performed without adjunct antimetabolites, ${ }^{10,12}$ whereas others have observed success rates of up to $87.5 \%$ when transconjunctival needling is associated with 5-fluouracil (5-FU) or mitomycin-C (MMC). ${ }^{16-19}$

However, the long-term follow-up of patients developing EBs has not been sufficiently investigated. The purpose of this study is to evaluate the IOP control of eyes that developed an EB following trabeculectomy.

\section{Materials and methods}

Between January 1994 and December 1995, 282 trabeculectomies were performed at the Glaucoma Service of University of Campinas, Brazil. Among the 282 eyes, 25 (8.9\%) developed EBs and were randomized (as soon as the diagnosis was made) to medical treatment with 
aqueous humor suppressors or transconjunctival needling without adjunct antimetabolites. The EBs were diagnosed $5.2 \pm 3.1$ weeks after surgery in the medical treatment group and $4.1 \pm 1.1$ weeks after guarded filtration procedure in the group undergoing the needling procedure $(P=0.679)$. The methods and the results of this prospective, randomized study have been previously reported. ${ }^{10}$ The needling procedure was performed by the same ophthalmologist (VPC) as described by Pederson and Smith, ${ }^{11}$ with a few modifications. A lid speculum was placed between the lids. Under topical anaesthesia, balanced salt solution was injected subconjunctivally adjacent to the EB through a 27 -gauge needle attached to a $1 \mathrm{ml}$ syringe. The same 27-gauge needle was used to transfixate and open a $2 \mathrm{~mm}$ incision in the fibrous wall of the EB. The needle was then withdrawn, topical antibiotic drops were instilled, and a patch was applied to the eye for $24 \mathrm{~h}$.

Among the 25 patients who developed EBs and were included in the previous study, ${ }^{10} 21$ were followed for at least 6 months at the University of Campinas. Four of the 25 patients did not return for follow-up and were excluded from the study. In order to evaluate the longterm IOP control of eyes with EBs, a control group of 21 eyes which underwent trabeculectomy and did not develop EBs was elected. This control group was retrospectively selected, was constituted by consecutive eyes that underwent trabeculectomy during the same period and by the same surgeons, and was not included in the previous publication. ${ }^{10}$

Mean IOP and mean number of antiglaucoma medications in each group were compared using analysis of variance (ANOVA). Success was defined as IOP $<21 \mathrm{mmHg}$ with or without antiglaucoma medications. Failure was defined as IOP $>21 \mathrm{mmHg}$ or when a new glaucoma surgery was indicated to improve IOP control. Between-group comparisons were performed using the $\chi^{2}$ test or the Fisher's exact test for categorical data. Kaplan-Meier statistical survival analysis was performed to compare the groups. $P$-values of less than 0.05 were considered statistically significant. This study was approved by the Ethics Committee of the University of Campinas.

\section{Results}

Among the 21 eyes that developed EBs, 12 (57\%) had undergone transconjunctival needling and nine (43\%) had received medical treatment. Table 1 displays the demographics of all groups. There was no statistically significant difference regarding the mean age, and the distribution of gender and race among the groups. Adjunct antimetabolites (MMC or 5-FU) had been used in five eyes (42\%) of the needling group, four eyes (44\%) of the medical treatment group, and five eyes (24\%) of the control group $(P=0.49)$. Mean follow-up was $30.0 \pm 14.0$ months for the needling group, $33.3 \pm 18.5$ months for the medical treatment group, and $37.4 \pm 2.6$ months for the control group $(P=0.19)$. Furthermore, the number of eyes with previous intraocular surgery was similar between the groups $(P=0.64)$.

Table 2 lists the preoperative and postoperative IOP measurements in each group at different time intervals. Mean IOP was significantly higher in the needling group $(19.8 \pm 4.1 \mathrm{mmHg})$ than in the medical treatment $(14.0 \pm 4.7 \mathrm{mmHg})$ and control groups $(12.4 \pm 3.4 \mathrm{mmHg})$ $(P<0.0001)$ six months following trabeculectomy. There was no statistically significant difference in mean IOP values between the groups at all other time intervals.

Table 3 compares the mean number of medications used in all groups during follow-up. Preoperatively, the mean number of medications used by the three groups did not differ statistically. From the sixth postoperative month on, patients developing EBs required significantly more antiglaucoma medications than the control group. Patients who required new surgical interventions to

Table 1 Demographic characteristics

\begin{tabular}{|c|c|c|c|c|}
\hline & $\begin{array}{l}\text { Needling group } \\
\quad(\mathrm{n}=12)\end{array}$ & $\begin{array}{l}\text { Medical treatment } \\
\text { group }(\mathrm{n}=9)\end{array}$ & $\begin{array}{l}\text { Control group } \\
\quad(\mathrm{n}=21)\end{array}$ & $\mathrm{P}$ \\
\hline Age (years) & $66.5 \pm 10.1$ & $66.6 \pm 19.3$ & $62.6 \pm 19.0$ & 0.75 \\
\hline Gender & F 3/M 9 & F 2/M 7 & F $10 / \mathrm{M} 11$ & 0.27 \\
\hline Race & W 5/B 7 & W 6/B 3 & W 16/B 5 & 0.13 \\
\hline \multirow[t]{2}{*}{ Diagnosis } & POAG 8 & POAG 6 & POAG 15 & 0.97 \\
\hline & CACG 4 & CACG 3 & CACG 6 & \\
\hline \multirow[t]{3}{*}{ Surgery } & TREC 7 & TREC 5 & TREC 16 & 0.49 \\
\hline & TRECM 5 & TRECM 3 & TRECM 4 & \\
\hline & & TRECF 1 & TRECF 1 & \\
\hline Number of eyes with previous intraocular surgeries & 3 & 2 & 3 & 0.64 \\
\hline
\end{tabular}

F, female; M, male; W, white; B, black; POAG, primary open-angle glaucoma; CACG, chronic angle-closure glaucoma; TREC, trabeculectomy without antimetabolites; TRECF, trabeculectomy with 5-FU; TRECM, trabeculectomy with MMC. 
Table 2 Mean IOP ( $\mathrm{mmHg}$ ) in each group at all time intervals

\begin{tabular}{lcccc}
\hline & Needling group & Medical treatment group & Control group & P \\
\hline Preoperative IOP & $24.00 \pm 3.01(n=12)$ & $26.55 \pm 8.88(n=9)$ & $24.42 \pm 4.98(n=21)$ & 0.94 \\
6 months & $19.81 \pm 4.07(n=12)$ & $14.00 \pm 4.71(n=9)$ & $12.38 \pm 3.39(n=21)$ & $<0.0001^{*}$ \\
1 year & $17.5 \pm 4.72(n=7)$ & $15.00 \pm 4.50(n=7)$ & $13.20 \pm 3.62(n=20)$ & 0.06 \\
2 years & $17.33 \pm 4.96(n=6)$ & $13.75 \pm 4.11(n=4)$ & $12.89 \pm 3.60(n=19)$ & 0.07 \\
3 years & $10.33 \pm 7.63(n=3)$ & $14.33 \pm 4.50(n=3)$ & $14.38 \pm 3.12(n=18)$ & 0.27 \\
4 years & $14(n=1)$ & $14.66 \pm 4.04(n=3)$ & $13.50 \pm 2.36(n=16)$ & \\
\hline
\end{tabular}

${ }^{*} P<0.05$ (ANOVA).

Bold represents $P<0.05$.

Table 3 Mean number of medications used in each group at all time intervals

\begin{tabular}{lcccc}
\hline & Needling group & Medical treatment group & Control group & P \\
\hline Preoperative & $2.41 \pm 0.79(n=12)$ & $2.80 \pm 0.60(n=9)$ & $2.31 \pm 1.50(n=21)$ & 0.29 \\
6 months & $2.27 \pm 0.46(n=12)$ & $2.11 \pm 0.60(n=9)$ & $0.20 \pm 0.69(n=21)$ & $<\mathbf{0 . 0 0 0 0 1}^{*}$ \\
1 year & $2.28 \pm 0.48(n=7)$ & $2.28 \pm 0.95(n=7)$ & $0.35 \pm 0.93(n=20)$ & $<\mathbf{0 . 0 0 0 0 1}^{*}$ \\
2 years & $2.66 \pm 0.51(n=6)$ & $2.25 \pm 0.50(n=4)$ & $0.73 \pm 1.24(n=19)$ & $\mathbf{0 . 0 0 1}^{*}$ \\
3 years & $3.33 \pm 0.57(n=3)$ & $2.66 \pm 0.57(n=3)$ & $1.05 \pm 1.25(n=18)$ & $\mathbf{0 . 0 0 6 ^ { * }}$ \\
4 years & $4(n=1)$ & $2.66 \pm 0.57(n=3)$ & $1.37 \pm 1.25(n=16)$ & \\
\hline
\end{tabular}

${ }^{*} P<0.05$ (ANOVA).

Bold represents $P<0.05$.

lower the IOP were excluded from these analyses from the time surgery was performed.

Among the patients with EBs undergoing needling, the success rates were $100 \%$ at 6 months, $58 \%$ at 1 year, $54 \%$ at 2 years, $37 \%$ at 3 years, and $14 \%$ at 4 years. Among the patients with EBs undergoing medical treatment, the success rates were $100 \%$ at 6 months, $100 \%$ at 1 year, $71 \%$ at 2 years, and $67 \%$ at 3 and 4 years. In the control group, the percentage of patients classified as successful were $100 \%$ at 6 months, $95 \%$ at 1 year, $90 \%$ at 2 years, $86 \%$ at 3 years, and $81 \%$ at 4 years.

Kaplan-Meier survival curves comparing the needling and the medical treatment groups demonstrated a tendency for a higher risk of failure in the needling group $(P=0.054)$ (Figure 1). When the control group was compared to the groups developing EBs, Kaplan-Meier analysis showed a significantly higher chance of failure in both the needling and the medical treatment groups $(P<0.0001)$ (Figure 1).

\section{Discussion}

The primary management of bleb encapsulation has been to restart medical therapy, with several studies suggesting that this approach is highly successful in maintaining adequate IOP control. ${ }^{7-10,15}$ We performed a study that enrolled 25 patients with EBs, ${ }^{10}$ who were randomized to either needling $(n=14)$ or medical treatment with aqueous humor suppressants $(n=11)$. After a mean follow-up of 9.6 months, medical treatment alone was successful in maintaining IOP $<21 \mathrm{mmHg}$ in 10 patients (91\%), whereas needling alone was successful

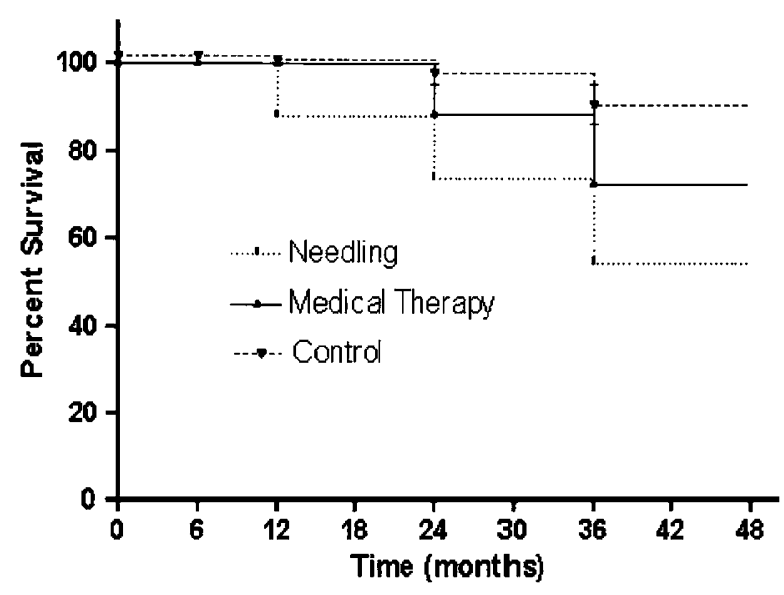

Figure 1 Kaplan-Meier survival curves.

in one patient $(7 \%)(P=0.00003)$. In the needling group, 93\% of the eyes required aqueous humor suppressants to improve IOP control. At the last follow-up examination, there was no statistically significant difference between the mean number of medications in both groups $(P=0.79)$.

Recently, Mandal ${ }^{15}$ conducted a retrospective study of 503 patients who had undergone trabeculectomy performed over a 5-year period, and had been followed up for at least 1 year. The authors identified 18 eyes (4\%) that developed EBs. MMC-augmented $(0.4 \mathrm{mg} / \mathrm{ml}$ for $3 \mathrm{~min}$ ) excisional bleb revision was performed in cases of failed medical therapy. In all, 15 (83\%) of 18 eyes responded to conservative management and three eyes (17\%) required MMC-augmented excisional bleb 
revision. The mean IOP reduced from $30.4 \pm 11.7$ to $14.2 \pm 4.2 \mathrm{mmHg}$ after a mean follow-up of $37.6 \pm 11.4$ months in the conservative management group, while it reduced from $37.3 \pm 23.4$ to $12.0 \pm 4.4 \mathrm{mmHg}$ after a mean follow-up of $42 \pm 6$ months in the surgically treated group. The author concluded that conservative management was very effective in the treatment of encapsulated filtering blebs and that intraoperative use of MMC was a safe and effective adjunct in excisional bleb revision in cases where conservative management had failed.

On the other hand, other investigators suggested that EBs might be a risk factor for surgical failure. ${ }^{20-22}$ Koller et $a l^{20}$ retrospectively assessed the charts of 388 trabeculectomies performed between 1992 and 1994 and concluded that pseudophakia and EBs were the main risk factors for surgical failure. Gutierrez et $a l^{21}$ prospectively studied 90 eyes that underwent trabeculectomy and were followed-up for at least 3 months. The authors found clinical characteristics of encapsulation in 29 eyes (32\%) and observed that EBs were significantly associated with an increased failure rate $(21 \%)$ when compared to normal blebs (5\% failure rate) $(P<0.001)$.

According to data from the Advanced Glaucoma Intervention Study, ${ }^{22}$ the 4 -week postoperative mean IOP was higher in eyes with than without EBs. With the resumption of medical treatment, the two means converged after 1 year. Richter et al ${ }^{1}$ reported a similar difference between the IOPs of eyes that developed EBs and eyes that did not develop this complication during the first few weeks after trabeculectomy. At 1 week, mean IOP was $2.3 \mathrm{mmHg}$ higher in eyes with EBs, with the difference increasing to $10.2 \mathrm{mmHg}$ at 3 weeks, and decreasing to $3.0 \mathrm{mmHg}$ at final examination.

However, there are few studies in the literature evaluating the long-term follow-up of eyes that developed EBs after filtering surgery. Despite the small number of patients included in each group, our findings confirm that EBs are associated with a greater risk for surgical failure. We observed that $100 \%(12 / 12)$ of the eyes that underwent needling needed hypotensive medication for IOP control at the 6th month of follow-up. Although all patients achieved IOP control at the 6th month, the mean IOP was higher in the needling group than in the medical treatment and control groups $(P<0.0001)$. Among the 12 eyes that underwent needling, five (42\%) maintained IOPs above $21 \mathrm{mmHg}$ and needed a new surgical intervention during the first year of follow-up. Among the nine eyes that received aqueous suppressants as initial treatment, none required a new surgical intervention during the first year, indicating that IOPs are adequately controlled with medication early after the development of EBs. However, patients who did not present EBs required less medication for IOP control during all the follow-up (Table 3). Furthermore, KaplanMeyer survival curves demonstrated significantly higher risks for failure in eyes developing EBs, regardless of the type of treatment they were submitted to (needling or medical therapy). After 48 months of follow-up, patients with EBs receiving medical treatment had a $33 \%$ chance of failure, whereas the needling group and the control group had 86 and $9 \%$ chance of failure, respectively $(P<0.0001)$ (Figure 1$)$.

It is possible that an invasive procedure, such as needling, can induce a strong fibroblastic proliferation, quickly scarring the openings of the capsule's wall, hindering the development of a functioning bleb, and probably compromising future procedures that could be necessary. This may explain the higher need for new surgeries in this group during the early postoperative period. Even those eyes that presented EBs and received antiglaucoma medication as initial treatment showed a higher risk for surgical failure. We hypothesize that the long-term use of aqueous humor suppressants may affect the bleb's function, ${ }^{8}$ by decreasing the amount of aqueous flowing to the subconjunctival space. Finally, it is possible that the wound healing process that leads to the development of an EB is per se associated with an increased proliferation of fibroblasts, facilitating the scarring of the filtration site.

Some authors have reported that multiple needle revisions are sometimes required. ${ }^{11-13}$ It is possible that repeated needling might have improved our results. However, repeated needling procedures without antimetabolites may stimulate fibroblastic proliferation even more, and may increase the risk of endophthalmitis, choroidal detachment, and shallow anterior chamber. Some authors suggested that needling with adjunctive 5FU or MMC may inhibit fibroblastic proliferation, increasing the success rates of this procedure. ${ }^{12,13}$ BenSimon and Glovinsky ${ }^{19}$ reported a $76 \%$ success rate of needling with MMC in 41 eyes 6 months after the procedure. Mardelli et $a l^{16}$ reviewed the charts of 62 eyes with failed filtering blebs that underwent needle revision using MMC and observed that the IOP decreased from $24.1 \pm 6.4 \mathrm{mmHg}$ before needling to $11.5 \pm 4.8 \mathrm{mmHg}$ at the last follow-up. Recently, Iwach et $a l^{17}$ reported a $71.6 \%$ success rate (IOP reduction of at least $30 \%$ without medication) of needling with transconjunctival application of MMC with a sponge in 42 eyes after 2 years of follow-up. However, all these series included eyes with flat, failing blebs, which may respond differently to the procedure when compared to eyes with EBs. Allen $e t a l^{18}$ performed the only retrospective study investigating the efficacy and safety of needling with adjunctive 5-FU in eyes with EBs. The authors reviewed a series of 32 eyes followed for $10.7 \pm 2.9$ months and 
observed a success rate (IOP $\leq 18 \mathrm{mmHg}$ with or without medication) of $87 \%$. Nevertheless, there is no long-term, prospective study investigating the success rates of needling with adjunctive 5-FU or MMC in eyes with EBs.

Our findings indicate that EBs are associated with an increased risk for surgical failure. Long-term, randomized studies are needed to investigate whether needling with 5-FU or MMC may enhance the survival of EBs.

\section{References}

1 Richter CU, Shingleton BJ, Bellows AR, Hutchinson BT, $\mathrm{O}^{\prime}$ Connor T, Brill I. The development of encapsulated filtering blebs. Ophthalmology 1988; 95: 1163-1168.

2 Feldman RM, Gross RL, Spaeth GL, Steinmann WC, Varma $\mathrm{R}$, Katz LJ et al. Risk factors for development of Tenon's capsule cysts after trabeculectomy. Ophthalmology 1989; 96: 336-341.

3 Oh Y, Katz LJ, Spaeth GL, Wilson RP. Risk factors for the development of encapsulated filtering blebs. The role of surgical glove powder and 5-fluorouracil. Ophthalmology 1994; 101: 629-634.

4 Feldman RM, Gross RL, Wilson RP, Spaeth GL, Varma R, Eagle RC. Encapsulated filtering blebs. Arch Ophthalmol 1987; 105: 1589.

5 Starita RJ, Fellman RL, Spaeth GL, Poryzees EM, Greenidge KC, Traverso CE. Short- and long-term effects of postoperative corticosteroids on trabeculectomy. Ophthalmology 1985; 92: 938-946.

6 Van Buskirk EM. Cyst of Tenon's capsule following filtration surgery. Am J Ophthalmol 1982; 94: 522-527.

7 Sherwood MB, Spaeth GL, Simmons ST, Nichols DA, Walsh AM, Steinmann WC et al. Cyst of Tenon's capsule following filtration surgery - medical management. Arch Ophthalmol 1987; 105: 1517-1521.

8 Scott DR, Quigley HA. Medical management of a high bleb phase after trabeculectomies. Ophthalmology 1988; 95: 1169-1173.

9 Shingleton BJ, Ritcher CU, Bellows AR, Hutchinson T. Management of encapsulated filtering blebs. Ophthalmology 1990; 97: 63-68
10 Costa VP, Correa MM, José NK. Needling versus medical treatment in encapsulated blebs - a randomized, prospective study. Ophthalmology 1997; 104: 1215-1220.

11 Pederson JE, Smith SG. Surgical management of encapsulated filtering blebs. Ophthalmology 1985; 92: 955-958.

12 Ewing RH, Stamper RL. Needle revision with and without 5 -fluorouracil for the treatment of failed filtering blebs. Am J Ophthalmol 1990; 110: 254-259.

13 Shin DH, Juzych MS, Khatana AK, Swendris RP, Parrow KA. Needling revision of failed filtering blebs with adjunctive 5-fluorouracil. Ophthalmic Surg 1993; 24: 242-248.

14 Potash SD, Ritch R, Liebmann J. Ocular hypotony and choroidal effusion following bleb needling. Ophtahlmic Surg 1993; 24: 270-280.

15 Mandal AK. Results of medical management and mitomycin C-augmented excisional bleb revision for encapsulated filtering blebs. Ophthalmic Surg Lasers 1999; 30: 276-284.

16 Mardelli PG, Lederer Jr CM, Murray PL, Pastor SA, Hassanein KM. Slit-lamp needle revision of failed filtering blebs using mitomycin C. Ophthalmology 1996; 103: 1946-1955.

17 Iwach AG, Delgado MF, Novack GD, Nguyen N, Wong PC. Transconjunctival mitomycin-C in needle revisions of failing filtering blebs. Ophthalmology 2003; 110: 734-742.

18 Allen LE, Manuchehri K, Corridan PG. The treatment of encapsulated trabeculectomy blebs in an out-patient setting using a needling technique and subconjunctival 5fluorouracil injection. Eye 1998; 12: 119-123.

19 Ben-Simon GJ, Glovinsky Y. Needle revision of failed filtering blebs augmented with subconjunctival injection of mitomycin C. Ophthalmic Surg Lasers Imaging 2003; 34 94-99.

20 Koller TL, Sturmer J, Gloor B. Risk factors for trabeculectomy failure. Klin Monatsbl Augenheilkd 1998; 213: $1-8$.

21 Gutierrez DE, Montero RM, Julve SMA, Galvez RA, Mencia GE. Incidence of encapsulated bleb after filtering surgery. Arch Soc Esp Oftalmol 2001; 76: 279-284.

22 Schwartz AL, Van Veldhuisen PC, Gaasterland DE, Ederer F, Sullivan EK, Cyrlin MN. The Advanced Glaucoma Intervention Study (AGIS): 5. Encapsulated bleb after initial trabeculectomy. Am J Ophthalmol 1999; 127: 8-19. 